\title{
LSM3, NDUFB3, and PTGS2 may be potential biomarkers for $B R C A 1$-mutation positive breast cancer
}

\author{
Kang $\mathrm{Hu}^{1}$, Fengjiao $\mathrm{Gan}^{1}$, Xue Wang ${ }^{1}$, Lin $\mathrm{Xu}^{2}$, Qiaoyuan $\mathrm{Wu}^{2}$, Ni Jiang ${ }^{2}$, \\ Suhong $\operatorname{Sun}^{1 *}$
}

1. Breast and Thyroid Disease Medical Center, Affiliated Hospital of Zunyi Medical University, Zunyi, GuiZhou province, 563003, China

2. Department of Oncology Laboratory, Affiliated Hospital of Zunyi Medical University, Zunyi, GuiZhou province, 563003, China

\begin{abstract}
Purpose: We aimed to find critical biomakers associated with BRCA1-mutation positive breast cancer. Methods: The data set E-MTAB-982 was downloaded from ArrayExpress database and the data were preprocessed using $R$ package Oligo. Differential expression analysis between BRCA1-mutation positive breast cancer patients and BRCA1-mutation positive healthy subjects were performed using limma package. Then, gene set enrichment analysis was conducted. We constructed the network for BRCA1, its related differentially expressed genes (DEGs), and the enriched Kyoto Encyclopedia of Genes and Genomes (KEGG) pathways. After that, survival analysis was performed based on the clinical data of breast cancer in TCGA database. Finally, box diagram for key genes was drawn. Results: The network showed that LSM3, NDUFB3, GNPDA2, and PTGS2 were BRCA1 related DEGs. Furthermore, LSM3 was mainly enriched in RNA degradation pathway and spliceosome pathway. PTGS2 was enriched in arachidonic acid metabolism and VEGF signaling pathway. Survival analysis indicated that high expression of LSM3 indicated a poor prognosis of BRCA1-mutant breast cancer. Besides, box diagram showed that LSM3 was down-regulated in BRCA1-mutation positive breast cancer patients compared with that in BRCA1-mutation positive healthy subjects. Conclusions: LSM3, NDUFB3, and PTGS2 may be biomarkers in BRCA1-mutant breast cancer, and high expression of LSM3 may indicate a poor prognosis of BRCA1-mutant breast cancer.
\end{abstract}

Keywords: BRCA1-mutation; breast cancer; differentially expressed genes; pathways

Received: 23 ${ }^{\text {rd }}$ July 2020; Accepted: $28^{\text {th }}$ October 2020; Published: $31^{\text {st }}$ October 2020

\footnotetext{
* Corresponding author: Suhong Sun, Breast and Thyroid Disease Medical Center, Affiliated Hospital of Zunyi Medical University, No.149 DaLian Road, HuiChuan District, Zunyi City,GuiZhou Province, 563003, China. -mail: s5nt8579t@sina.com
} 


\section{Introduction}

Breast cancer is a common disease with high mortality and morbidity (1). It is expected that breast cancer accounts for $30 \%$ of all newly diagnosed female cancers (2). The signs of it include a change in the breast shape, a lump in the breast, a red scaly patch of skin and so on (3). Risk factors for this cancer include obesity, drinking alcohol, older age, family history and being female (4). The survival rates of breast cancer are poorer in developing countries (4). In spite of advances in therapeutic methods such as chemotherapy and surgical therapy, mortality related with breast cancer still increases in China (5), which is due to the difficulties in the finding of diagnostic and treatment methods for breast cancer. Furthermore, with the increasing morbidity and the trend of young age in recent years, breast cancer threatens the women's health (6). It is necessary to find more effective diagnostic and treatment targets for breast cancer.

It is reported that $B R C A 1$ and $B R C A 2$ were two major predisposing genes for breast cancer (7). The risk of breast cancer was increased in $B R C A 1$ and $B R C A 2$ mutation carriers (8). The lifetime risk of breast cancer in $B R C A 1$-mutation carriers is $85 \%$ (9). Thus, BRCA1 plays critical role in breast cancer development, and exploring the molecular mechanisms of BRCA1-mutant breast cancer may provide clues for the early diagnosis and treatment of the disease. Recent studies have reported some important genes associated with breast cancer. For instance, Liu et al. (10) identified that FOS, IL6 and FN1 might be regarded as treatment targets for breast cancer. ARID $4 A$ and $C D C 20$ may be used to predict the prognosis of breast cancer after chemotherapy (11). Wu et al. (12) indicated that ADAMTS1 might be involved in the development of breast cancer via regulating angiogenesis, and CCNG1 might play a key role in breast cancer progression through inhibition of cell proliferation. Although the former studies reported some key genes in breast cancer, there were few studies on the key potential target genes in breast cancer with BRCAl-mutantion. Therefore, it is needed to study critical target genes in BRCA1-mutation positive breast cancer. In our present study, the dataset E-MTAB-982 was downloaded and the data were preprocessed. Differential expression analysis between BRCA1-mutation positive breast cancer patients and $B R C A 1$-mutation positive healthy subjects was performed to identify the differentially expressed genes (DEGs). Then, gene set enrichment analysis (GSEA) was conducted. We constructed the network for $B R C A 1$, its related DEGs, and the enriched Kyoto Encyclopedia of Genes and Genomes (KEGG) pathways. After that, survival analysis was performed based on the RNA-Seq and clinical data in The Cancer Genome Atlas (TCGA) database. Finally, box diagram for key genes was drawn. We aimed to find critical target genes associated with $B R C A 1$-mutant breast cancer.

\section{Methods}

\section{Data source and data preprocessing}

The dataset E-MTAB-982 was downloaded from ArrayExpress (13) database (https://www. ebi.ac.uk/arrayexpress/). The dataset included 10 lymphocyte samples obtained from breast cancer patients with BRCA1-mutation (cancer group) and 11 lymphocyte samples from normal subjects with BRCA1-mutation (control group) (The samples with ovarian carcinoma, or mixed samples with breast carcinoma and ovarian carcinoma were excluded). The platform was Affymetrix GeneChip Human Gene 1.0 ST Array [HuGene-1_0-st-v1].

Background correction and data normalization (including conversion of original data format, supplement of missing value, background correction, and data standardization) were conducted for the original CEL data using $\mathrm{R}$ package 
Oligo (14) (version 1.46.0, http://bioconductor. org/help/search/index.html?q=oligo/). Annotation for probes was performed using platform annotation files, and probes not matching to gene symbol were removed. For different probes matching to the same gene, the mean values of different probes were regarded as the final expression value of this gene.

\section{Differential expression analysis}

Differential expression analysis between cancer group and control group were performed using limma package (15) (version 3.38.3, http://www. bioconductor.org/packages/2.9/bioc/html/limma. html). Genes with $\mathrm{p}$ value $<0.05$ and $\mid \log$ fold change (FC) $\mid \geq 0.585$ were considered as DEGs.

\section{GSEA and network construction}

Pearson correlation coefficients between $B R C A 1$ and each gene were calculated using GSEA software (V3.0) (16). In detail, the parameters were set as follows: gene sets database selected c2.cp. kegg.v6.2.symbols.gmt; the expression value of $B R C A 1$ was set as the phenopyte labels; Pearson was selected for metric for ranking genes to calculate Pearson correlation coefficients between BRCAl and each gene. The correlation coefficients were sorted in descending order (gene list sorting mode: real; gene list ordering mode: descending), and the KEGG pathway enrichment analysis was performed (17). Genes with $|r| \geq 0.3$ and $\mathrm{p}$ value $<0.05$ were considered significantly correlated with $B R C A 1$. Pathways with $\mid$ Normalized Enrichment Score (NES) $\mid>1$, NOM $p$ value $<0.05$, and false discovery rate (FDR)-corrected q value $<0.25$ were regarded as significantly enriched pathways. Intersections of genes significantly correlated with $B R C A 1$ and DEGs were set as BRCA1 related DEGs. The pathways significantly enriched by these genes were screened.

Based on the BRCA1, obtained BRCA1 related DEGs, and their enriched KEGG pathways, the interaction network was constructed using Cytoscape (version 3.3.0) (18).

\section{Survival analysis based on data from TCGA database}

To investigate the effects of BRCAl related DEGs on the prognosis of breast cancer, we downloaded the RNA-Seq and clinical data of breast cancer from TCGA database (https://tcga-data.nci.nih.gov/). The samples that had clinical data were selected, and 1067 samples were finally included. Additionally, the sample information was filtered and the samples with survival time $<1$ month were removed. The DEGs corresponding samples were retained. Survival curves were generated using the Kaplan-Meier method, and log-rank test was used to assess the differences between the high- and low-expression groups (based on median grouping) using survival package (version 2.42.6) (19) in R. Survival curve was drawn and genes with $p<0.05$ were selected.

\section{Box diagram for key genes}

Box diagram for key genes was drawn according to their expression values in cancer and control groups, showing the difference of these genes in different groups. The $p$ values for comparison of expression in genes were analyzed using limma package (15) (version 3.38.3).

\section{Results}

\section{Differential expression analysis}

Samples expression matrix was obtained through data preprocessing. After differential expression analysis, 164 (88 up-regulated and 76 down-regulated) DEGs for cancer vs. control groups were obtained. The heatmap and volcano plot for DEGs between two groups are presented in Figure 1. There was no significant difference in the expression of BRCAl between two groups $(\log \mathrm{FC}=-0.213$, $\mathrm{p}$ value $=0.03)$. For the 

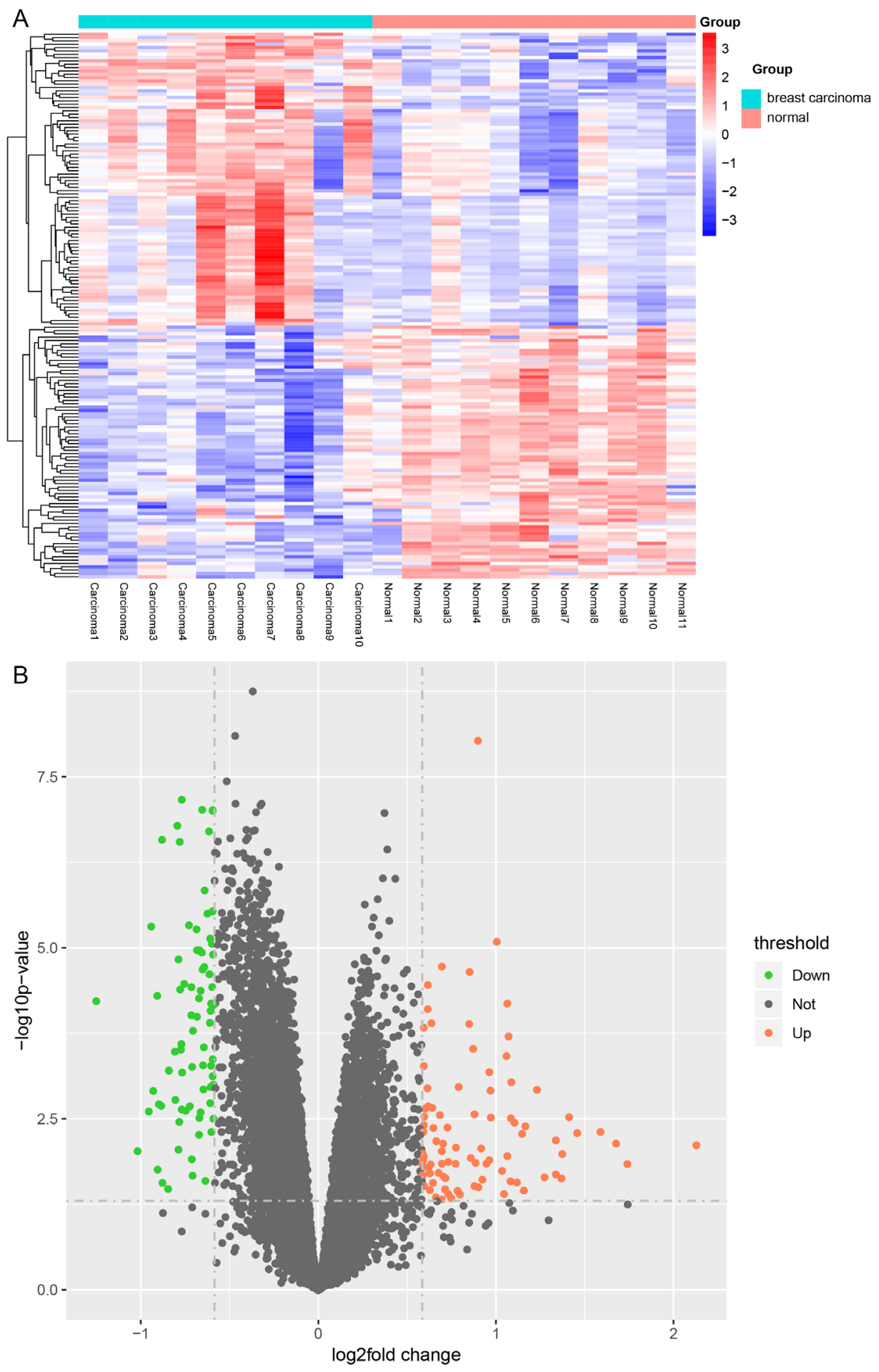

Fig. 1. The heatmap (A) and volcano plot (B) for differentially expressed genes (DEGs) between tumor and normal samples. 
subsequent analysis, $B R C A 1$ was also included in the DEGs list.

\section{GSE $A$ results}

The results showed that 47 positive pathways and 28 negative pathways were enriched by $B R C A 1$ related DEGs. In this analysis, BRCA1 was significantly enriched in ubiquitin mediated proteolysis pathway ( $\mathrm{p}$-value $<0.01$; q-value
$=0.01)$ (Figure 2). Table 1 shows the pathways enriched by leading subset genes that were positively and negatively correlated with $B R C A 1$.

\section{Network construction}

The network for $B R C A 1, B R C A 1$ related DEGs, and KEGG pathways enriched by $B R C A 1$ is presented in Figure 3. BRCA1 related DEGs included LSM3 homolog, U6 small nuclear RNA

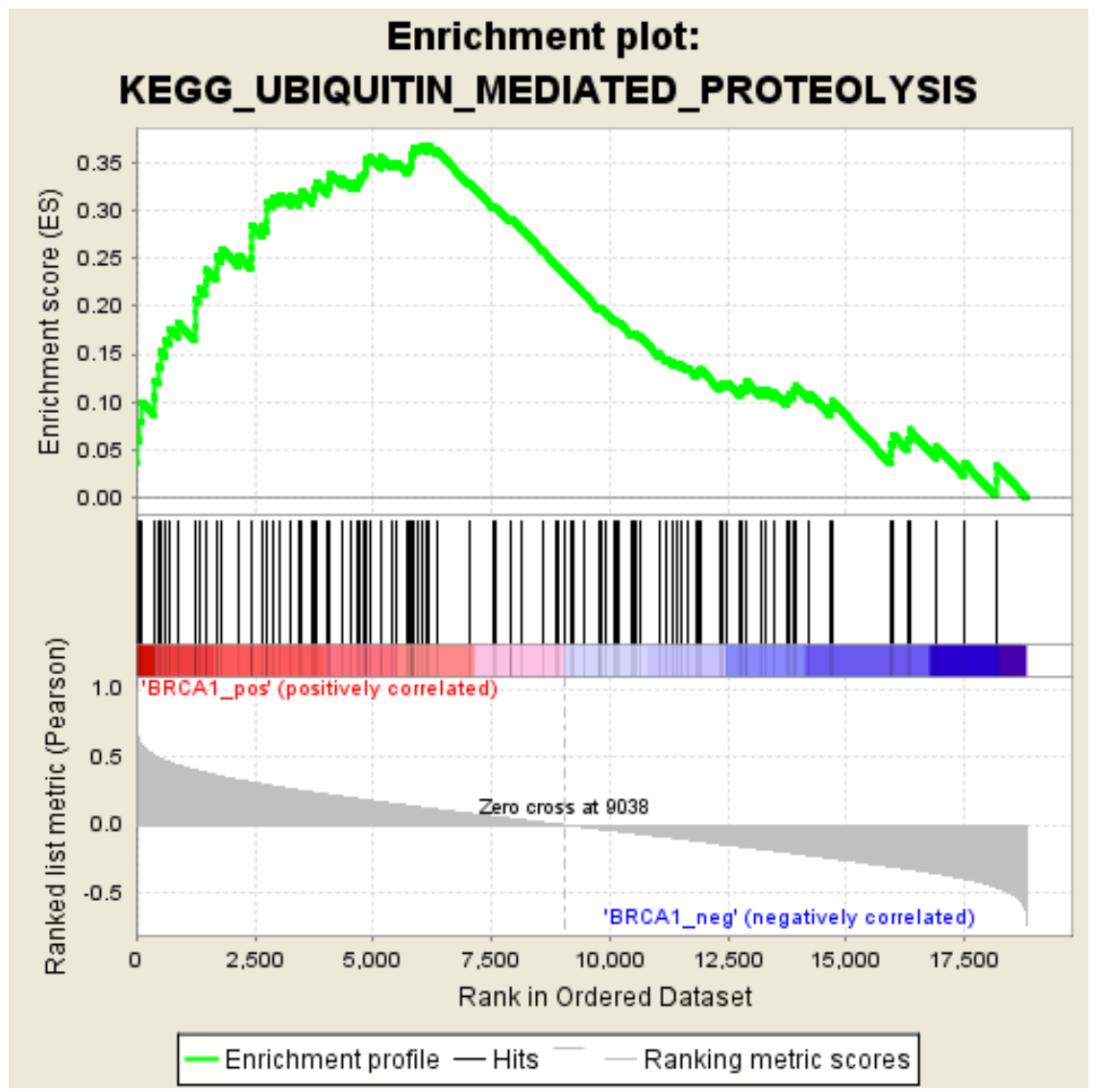

Fig. 2. The enrichment plot of ubiquitin mediated proteolysis pathway. The first part of the diagram is enrichment score line chart, which shows the ES value in each position when the analysis is calculated along the ranking list. The score at the highest peak (the furthest vertical distance from 0.0 ) is the ES value of the gene set. The second part of the diagram is that lines are used to mark the location of members of the gene set in the gene sequencing list, and the black lines represent the functional annotated gene set of current analysis for genes in the sequencing gene table. Leading edge subset is the corresponding genes from $(0,0)$ to green curve peak ES. The third part of the diagram is rank value of all genes after ranking. The genes corresponding to the red part of the heat map are positively related to $B R C A 1$, and the blue part is negatively related to $B R C A 1$. The grey area represents the Pearson correlation coefficient corresponding to each gene. 
Table 1 The pathways enriched by leading subset genes positively and negatively correlated with BRCA1.

\begin{tabular}{lcccccc}
\hline NAME & GENE & $\begin{array}{c}\text { RANK } \\
\text { METRIC } \\
\text { SCORE }\end{array}$ & $\begin{array}{c}\text { CORE } \\
\text { ENRICH- } \\
\text { MENT }\end{array}$ & $\begin{array}{c}\text { NES } \\
\text { NOM }\end{array}$ & $\begin{array}{c}\text { FDR } \\
\text { p-val }\end{array}$ \\
q-val \\
\hline KEGG_ALZHEIMERS_DISEASE & NDUFB3 & 0.42 & Yes & 1.39 & 0.03 & 0.14 \\
\hline $\begin{array}{l}\text { KEGG_AMINO_SUGAR_AND_NUCLEOTIDE_ } \\
\text { SUGAR_METABOLISM }\end{array}$ & GNPDA2 & 0.39 & Yes & 2.21 & $<0.01$ & $<0.01$ \\
\hline KEGG_ARACHIDONIC_ACID_METABOLISM & PTGS2 & -0.34 & Yes & -2.05 & $<0.01$ & $<0.01$ \\
\hline KEGG_HUNTINGTONS_DISEASE & NDUFB3 & 0.42 & Yes & 1.71 & $<0.01$ & 0.02 \\
\hline KEGG_OXIDATIVE_PHOSPHORYLATION & NDUFB3 & 0.42 & Yes & 1.84 & $<0.01$ & 0.01 \\
\hline KEGG_PARKINSONS_DISEASE & NDUFB3 & 0.42 & Yes & 1.45 & 0.02 & 0.10 \\
\hline KEGG_RNA_DEGRADATION & LSM3 & 0.36 & Yes & 2.53 & $<0.01$ & $<0.01$ \\
\hline KEGG_SPLICEOSOME & LSM3 & 0.36 & Yes & 2.90 & $<0.01$ & $<0.01$ \\
\hline KEGG_UBIQUITIN_MEDIATED_PROTEOLYSIS & BRCA1 & 1.00 & Yes & 1.85 & $<0.01$ & 0.01 \\
\hline KEGG_VEGF_SIGNALING_PATHWAY & PTGS2 & -0.34 & Yes & -1.77 & $<0.01$ & 0.02 \\
\hline
\end{tabular}

NES: ES value after normalization; NOM p-val: p-value; FDR q-val: q-value; CORE ENRICHMENT: Yes represents that the gene is located in the leading subset of the entire set of genes; RANK METRIC SCORE: correlation coefficient between BRCA1 and other genes.
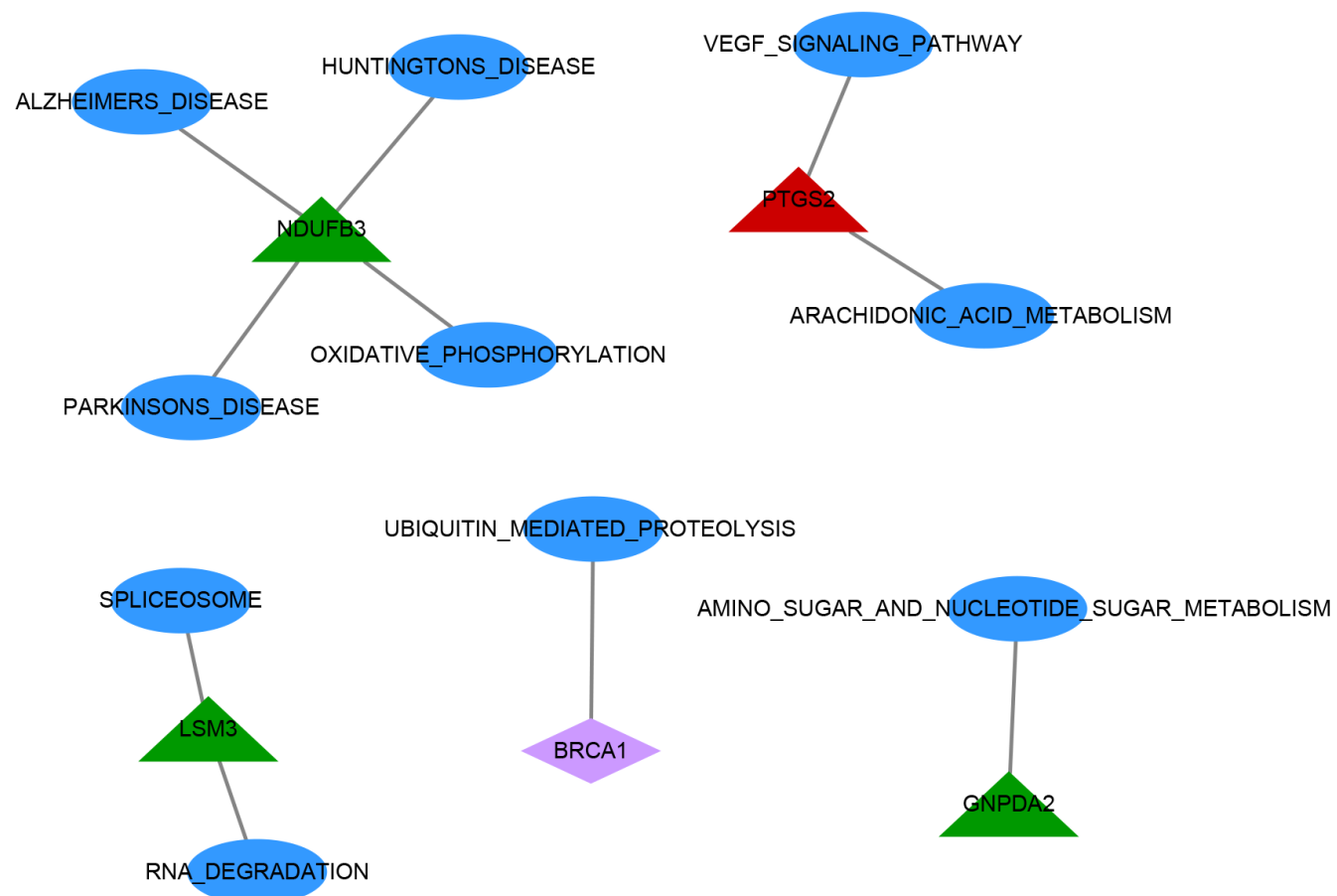

Fig. 3. The network for $B R C A 1$, its related differentially expressed genes (DEGs), and the enriched KEGG pathways. Blue oval: KEGG pathway; pink diamond: BRCA1 gene; red triangle: up-regulated DEGs; green triangle: down-regulated DEGs. 
and mRNA degradation associated (LSM3), NADH: ubiquinone oxidoreductase subunit B3 (NDUFB3), glucosamine-6-phosphate deaminase 2 (GNPDA2), and prostaglandin-endoperoxide synthase 2 (PTGS2). LSM3 was significantly enriched in RNA degradation pathway (p-value $<0.01 ;$ q-value $<0.01)$ and spliceosome pathway (p-value $<0.01 ;$ q-value $<0.01$ ). NDUFB 3 was enriched in Alzheimers disease ( $\mathrm{p}$-value $=0.03 ; \mathrm{q}$-value $=0.14)$, Huntingtons disease ( $\mathrm{p}$-value $<0.01 ; \mathrm{q}$-value $=0.02)$, oxidative phosphorylation (p-value $<0.01 ; \mathrm{q}$-value $=$ 0.01 ), and Parkinsons disease ( $\mathrm{p}$-value $=0.02$; $\mathrm{q}$-value $=0.10) . P T G S 2$ was enriched in arachi- donic acid metabolism (p-value $<0.01$; q-value $<0.01$ ), and VEGF signaling pathway ( $\mathrm{p}$-value $<0.01$; q-value $=0.02$ ). GNPDA2 was enriched in amino sugar and nucleotide sugar metabolism (p-value $<0.01 ;$ q-value $<0.01$ ).

\section{Survival analysis based on data from TCGA}

Based on the data from TCGA, survival analysis was performed for $B R C A 1$-related genes enriched in GSEA. LSM3 was associated with survival of breast cancer $(\mathrm{p}=0.01)$. A higher expression of $L S M 3$ indicated a worse prognosis. Survival curve for $L S M 3$ is shown in Figure 4.
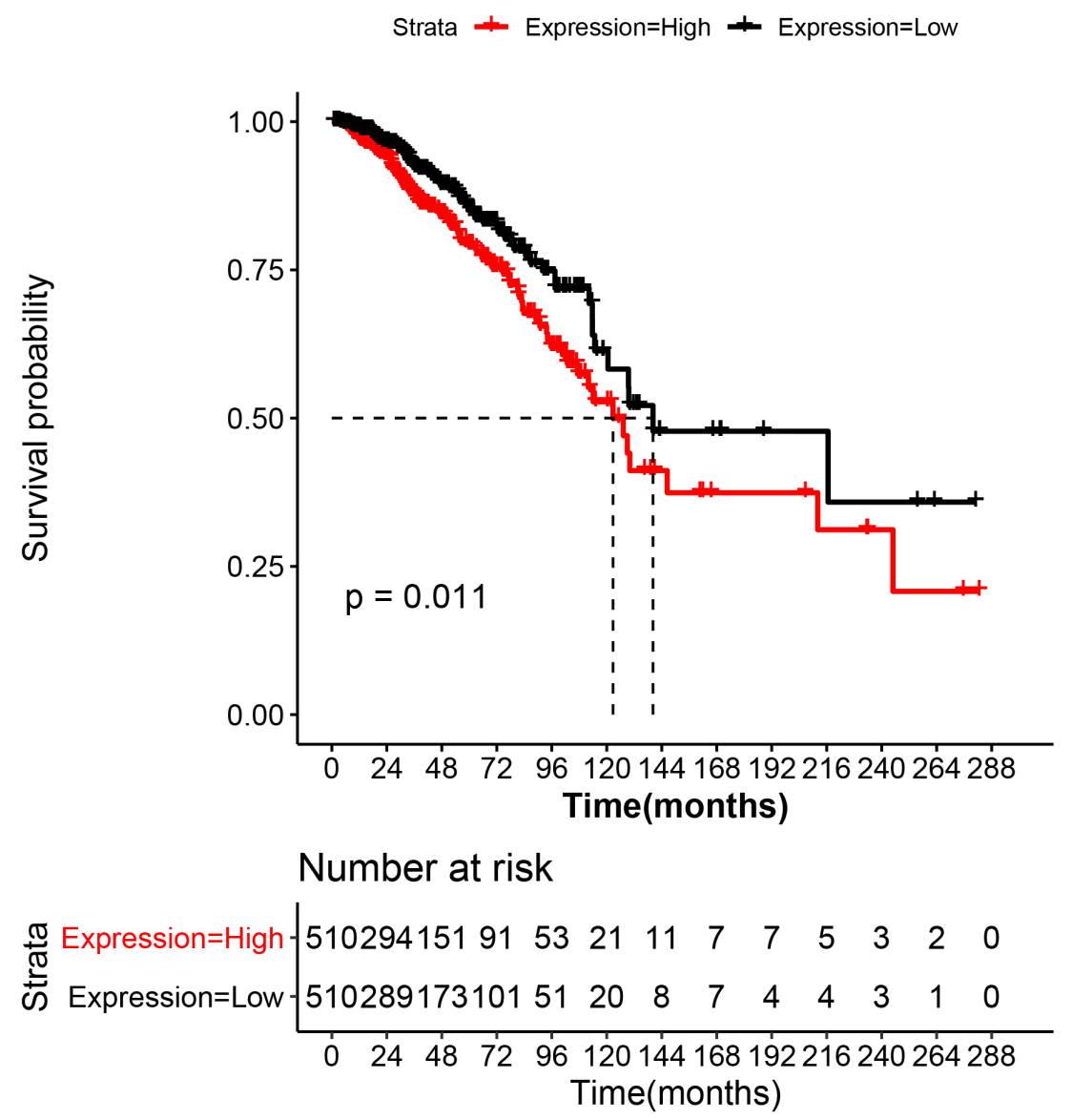

Fig. 4. Survival curve for LSM3 based on TCGA database. 


\section{Box diagram for key genes}

According to the expression values of $B R C A 1$ and LSM3 in cancer group and control group, the box diagrams of the two genes were drawn. The diagram showed that both BRCA1 and LSM3 were down-regulated in cancer group compared with that in control group (Figure 5).

\section{Discussion}

In this study, the dataset including 10 lymphocyte samples from breast cancer patients with BRCA1-mutation and 11 lymphocyte samples from normal persons with BRCA1-mutation were used for the analysis, and we aimed to identify critical target genes associated with BRCA1-mutation breast cancer. The constructed network showed that LSM3, NDUFB3, GNPDA2, and PTGS2 were BRCA1 related DEGs. Furthermore, LSM3 was mainly enriched in RNA degradation pathway and spliceosome pathway. PTGS2 was enriched in arachidonic acid metabolism and VEGF signaling pathway. Survival analysis based on the data in TCGA database indicated that LSM3 was associated with survival. Besides, box diagram showed that LSM3 was down-regulated in cancer group compared with that in control group.

It is reported that LSM3 proteins is physically related with some splicing factors (20). Splicing factor ratio is regarded as an index of tumor aggressiveness and epithelial-mesenchymal transition in breast cancer (21). A recent study has reported that single nucleotide polymorphism of LSM3 is significantly associated with colorectal cancer (22). GSEA result showed that NDUFB3 was enriched in oxidative phosphorylation pathway. Metabolic reprograming is an emerging hallmark of tumor biology. There is a group of cancers, including breast cancer, in which oxidative phosphorylation is down-regulated (23). Sotgia et al. (24) have suggested that high level of NDUFB3 predicts reduced overall survival in lung cancer patients. PTGS2 is undetectable in most normal tissues, and can be induced in response to stressors, such as inflammatory cytokines, hypoxia, and tumor promoters (25). It has been indicated that PTGS2 genetic variation is implicated in breast cancer susceptibility (26). Overexpression of PTGS2 in patients with breast cancer is associated with a worse prognosis (27). In HER2-negative breast cancer patients,
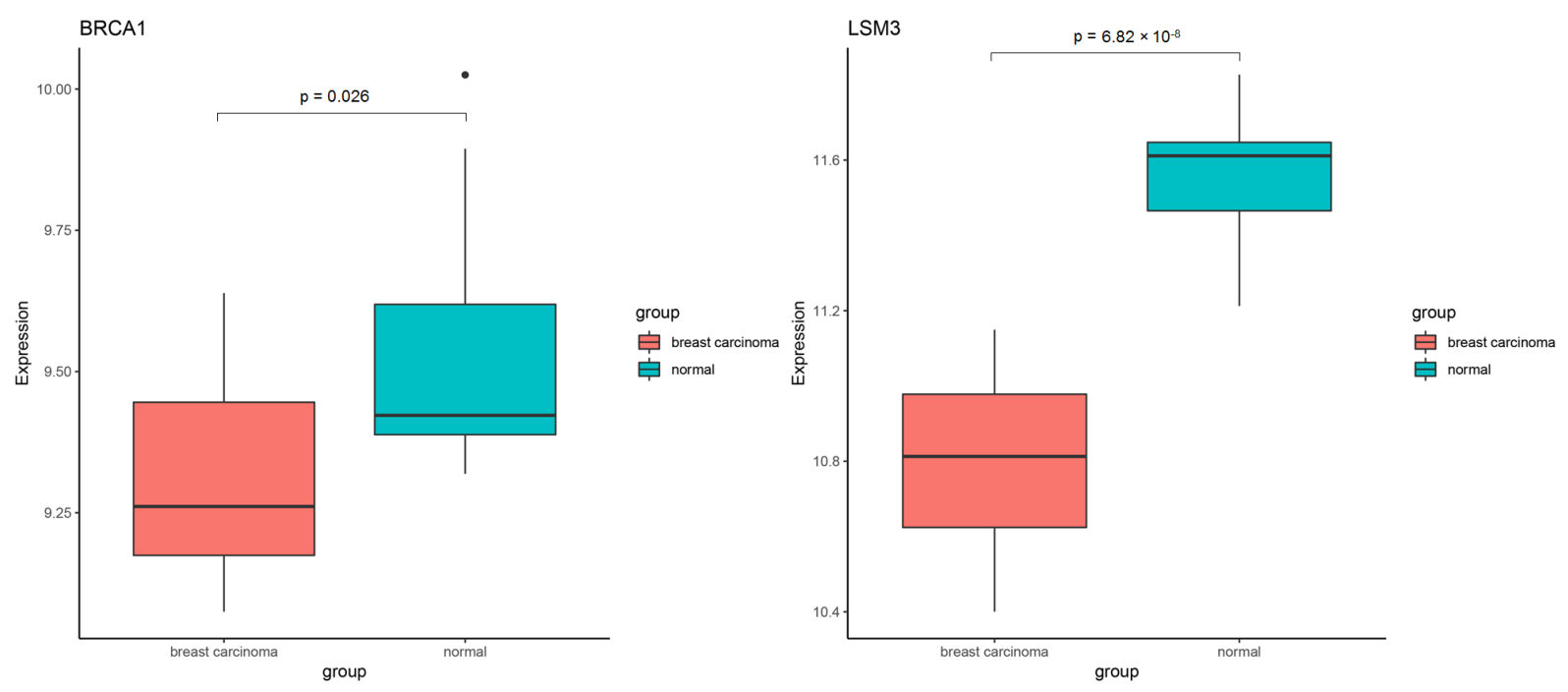

Fig. 5. Box diagram for $B R C A 1$ and $L S M 3$. 
the expression of PTGS2 predicts response to neoadjuvant celecoxib (28). Dossus et al. (26) indicated that PTGS2 polymorphisms were associated with the risk of breast cancer. To our knowledge, there is no studies about the association between GNPDA2 and breast cancers or other cancers. Taken together, we proposed that LSM3, NDUFB3 and PTGS2 may play significant roles in disease progression in breast cancer patients with BRCA1-mutation. The associations between GNPDA2 and breast cancers need further studies.

In addition, LSM3 was mainly enriched in RNA degradation pathway and spliceosome pathway. PTGS2 was enriched in arachidonic acid metabolism and VEGF signaling pathway. Studies have reported that RNA degradation is related with cell apoptosis $(29,30)$, an important biological process for cancer cell progression. Koedoot et al. (31) have suggested that spliceosome factors are drivers of breast cancer development. Deregulated spliceosome core machinery is important for mTOR blockade and autophagy in cancer cells (32). Arachidonic acid metabolism plays role in Stat5 activation in breast cancer cells (33). VEGF signaling pathway is crucial for inhibition of neoangiogenesis in breast cancer (34). Chun et al. (35) suggested that VEGF signaling pathway might be potential therapeutic target in obesity-related breast cancer. Therefore, we speculated that LSM3 may play roles in BRCA1-mutant breast cancer via RNA degradation pathway and spliceosome pathway, and PTGS2 may be involved in BRCA1-mutant breast cancer via arachidonic acid metabolism pathway and VEGF signaling pathway.

Furthermore, in our present study, survival analysis, through downloading RNA-Seq and clinical data from TCGA database, indicated that LSM3 was associated with the survival of breast cancer patients, and a higher expression of LSM3 indicated a worse prognosis. The box diagram showed that LSM3 was down-regulated in tumor samples compared with that in normal samples. This seems conflicting. But the role of gene in organism is complex. The differential expression of LSM3 in cancer and normal tissues is associated with the onset of cancer, and the survival prognosis of cancer is associated with the progression of cancer. We speculated that LSM3 is low in expression to combat certain aberrations in tumor cells, so patients with good prognosis may also have low expression of this genes, which needs to be further investigated. Therefore, the results were not conflicting. Combined with the above stated, we inferred that LSM3 may be an important prognostic factor for BRCA1-mutant breast cancer.

However, there was no validation experiments in this study. Additionally, in the dataset of E-MTAB-982, there are ovarian cancer associated data, nevertheless we did not compare breast cancer with and ovarian cancer. Investigating the correlations between the two cancers may bring some useful information in the future.

In conclusion, LSM3, NDUFB3 and PTGS2 may be potential biomarkers in BRCA1-mutant breast cancers. Furthermore, high expression of LSM3 may indicate a poor prognosis of BRCA1-mutant breast cancer according to the data in TCGA database. However, because of lacking of verification, further verification experiments are needed to confirm our present results.

\section{Abbreviations}

DEGs: differentially expressed genes; GSEA: gene set enrichment analysis; TCGA: The Cancer Genome Atlas; KEGG: Kyoto Encyclopedia of Genes and Genomes; FC: fold change; NES: Normalized Enrichment Score; LSM3: LSM3 homolog, U6 small nuclear RNA and mRNA degradation associated; NDUFB3: NADH: ubiquinone oxidoreductase subunit B3; GNPDA2: glucosamine-6-phosphate deaminase 2; PTGS2: prostaglandin-endoperoxide synthase 2 . 


\section{Acknowledgement}

This work was supported by National Natural Science Foundation of China, Program No. 81860469 , Project supported by the Zunyi Foundation for science and technology ,China, Grant Zunshi kehe HZ (2019) No.85; Grant Zunshi kehe HZ (2019) No.82.

\section{Authors' contributions}

S-H S and F-J G carried out the conception and design of the research and drafted the manuscript. S-H S participated in obtaining funding. $\mathrm{K} \mathrm{H}$ and $\mathrm{X} \mathrm{W}$ participated in the Acquisition of data. K H, Q-Y W and N J carried out the Analysis and interpretation of data. S-H S and L X participated in the design of the study and performed the statistical analysis.. All authors read and approved the final manuscript.

\section{Competing Interests}

The authors declare that they have no competing interests.

\section{References}

1. Güzey S, Aykan A, Avşar S, Yavan İ, Öztürk S. Recurrent Breast Cancer with Cutaneous Metastasis in the Late Term. Turkish Journal of Plastic Surgery. 2017;25(1):34+. DOI: 10.5152/TurkJPlastSurg.2016.1980

2. Siegel RL, Miller KD, Jemal A. Cancer Statistics, 2017. Ca Cancer J Clin. 2017;67(1):7-30. DOI: 10.3322/ caac. 21387

3. Cancer UK. Breast cancer symptoms. 2010.

4. McGuire, S. World Cancer Report 2014. Geneva, Switzerland: World Health Organization, International Agency for Research on Cancer, WHO Press, 2015. Advances in Nutrition: An International Review Journal. 2016;7(2):418-9. DOI: 10.3945/an.116.012211

5. Chen W, Zheng R, Baade PD, Zhang S, Zeng H, Bray F, et al. Cancer statistics in China, 2015. Ca Cancer J Clin. 2016;66(2):115-32. DOI: 10.3322/caac. 21338

6. Zhao X, Qu J, Sun Y, Wang J, Liu X, Wang F, et al. Prognostic significance of tumor-associated macrophages in breast cancer: a meta-analysis of the literature. Oncotarget. 2017;8(18):30576-86. DOI: 10.18632/oncotarget. 15736
7. Robson ME, Chappuis PO, Jaya S, Nora W, Jeff B, Goffin JR, et al. A combined analysis of outcome following breast cancer: differences in survival based on BRCA1/BRCA2 mutation status and administration of adjuvant treatment. Breast Cancer Res. 2003;6(1):R8. DOI: $10.1186 /$ bcr658

8. Kuchenbaecker KB, Hopper JL, Barnes DR, Phillips KA, Mooij TM, Roos-Blom MJ, et al. Risks of Breast, Ovarian, and Contralateral Breast Cancer for BRCA1 and BRCA2 Mutation Carriers. Jama. 2017 Jun 20;317(23):2402-16.

9. Anton-Culver H, Cohen PF, Gildea ME, Ziogas A. Characteristics of BRCA1 mutations in a population-based case series of breast and ovarian cancer. Eur J Cancer. 2000;36(10):1200-8. DOI: 10.1016/S09598049(00)00110-6

10. Liu X, Ma Y, Yang W, Wu X, Jiang L, Chen X. Identification of therapeutic targets for breast cancer using biological informatics methods. Mol Med Rep. 2015;12(2):1789. DOI: 10.3892/mmr.2015.3565

11. Zhao Y, Bing F. Screening of biomarkers for prediction of response to and prognosis after chemotherapy for breast cancers. Onco Targets Ther. 2016;9(Issue 1):2593-600. DOI: 10.2147/OTT.S92350

12. Wu D, Han B, Guo L, Fan Z. Molecular mechanisms associated with breast cancer based on integrated gene expression profiling by bioinformatics analysis. J Obstetrics \& Gynaecology. 2016;36(5):615-21. DOI: 10.3109/01443615.2015.1127902

13. Athar A, Fullgrabe A, George N, Iqbal H, Huerta L, Ali A, et al. ArrayExpress update - from bulk to single-cell expression data. Nucleic acids res. 2019 Jan 8;47(D1):D711-d5. DOI: 10.1093/nar/gky964

14. Carvalho BS, Irizarry RA. A framework for oligonucleotide microarray preprocessing. Bioinformatics. 2010;26(19):2363-7. DOI: 10.1093/bioinformatics/ btq431

15. Smyth GK. limma: Linear Models for Microarray Data. Bioinformatics \& Computational Biology Solutions Using R \& Bioconductor. 2011:397-420. DOI: 10.1007/0-387-29362-0_23

16. Subramanian A, Tamayo P, Mootha VK, Mukherjee S, Ebert BL, Gillette MA, et al. Gene set enrichment analysis: a knowledge-based approach for interpreting genome-wide expression profiles. Proc Natl Acad Sci U S A. 2005;102(43):15545-50. DOI: 10.1073/ pnas.0506580102

17. Kanehisa M, Goto S. KEGG: Kyoto Encyclopedia of Genes and Genomes. Nucleic Acids Res. 2000 Jan 1;28(1):27-30. DOI: 10.1093/nar/28.1.27

18. Shannon, P. Cytoscape: A Software Environment for Integrated Models of Biomolecular Interaction Networks. Genome Res. 2003;13(11):2498. DOI: 10.1101/ gr. 1239303

19. Therneau TM, Grambsch PM, Modeling Survival Data: 
Extending The Cox Model. 2000. DOI: 10.1007/978-14757-3294-8

20. Fromont-Racine M, Rain JC, Legrain P. Toward a functional analysis of the yeast genome through exhaustive two-hybrid screens. Nat Genet. 1997 Jul;16(3):277-82. DOI: $10.1038 / n g 0797-277$

21. Fici P, Gallerani G, Morel AP, Mercatali L, Ibrahim T, Scarpi E, et al. Splicing factor ratio as an index of epithelial-mesenchymal transition and tumor aggressiveness in breast cancer. Oncotarget. 2017;8(2):2423-36. DOI: 10.18632/oncotarget.13682

22. Xie N, Yao Y, Wan L, Zhu T, Liu L, Yuan J. Next-generation sequencing reveals lymph node metastasis associated genetic markers in colorectal cancer. Exp Ther Med. 2017;14(1):338-43. DOI: 10.3892/etm.2017.4464

23. Ashton TM, McKenna WG, Kunz-Schughart LA, Higgins GS. Oxidative phosphorylation as an emerging target in cancer therapy. Clin Cancer Res. 2018;24(11):2482-90. DOI: 10.1158/1078-0432.CCR17-3070

24. Sotgia F, Lisanti MP. Mitochondrial markers predict survival and progression in non-small cell lung cancer (NSCLC) patients: Use as companion diagnostics. Oncotarget. 2017;8(40):68095-107. DOI: 10.18632/oncotarget. 19677

25. Liu X-H, Kirschenbaum A, Yao S, Stearns ME, Holland JF, Claffey K, et al. Upregulation of vascular endothelial growth factor by cobalt chloride-simulated hypoxia is mediated by persistent induction of cyclooxygenase- 2 in a metastatic human prostate cancer cell line. Clin exp metastasis. 1999;17(8):687-94. DOI: 10.1023/A:1006728119549

26. Dossus L, Kaaks R, Canzian F, Albanes D, Berndt SI, Boeing $\mathrm{H}$, et al. PTGS2 and IL6 genetic variation and risk of breast and prostate cancer: results from the Breast and Prostate Cancer Cohort Consortium (BPC3). Carcinogenesis. 2010;31(3):455-61. DOI: 10.1093/carcin/bgp307

27. Festa-Vasconcellos JS, Piranda DN, Amaral LM, Indio-do-Brasil V, Koifman S, Vianna-Jorge R. Polymorphisms in cycloxygenase-2 gene and breast cancer prognosis: association between PTGS2 haplotypes and histopathological features. Breast cancer res treat. 2012;132(1):251-8. DOI: 10.1007/s10549-011-1828-0
28. De CP, Hamy AS, Lehmann-Che J, Scott V, Sigal B, Mathieu MC, et al. COX2/PTGS2 Expression Is Predictive of Response to Neoadjuvant Celecoxib in HER2-negative Breast Cancer Patients. Anticancer Res. 2018;38(3):1485. DOI: 10.21873/anticanres. 12375

29. Goswami BB, Michael K, Diana N, Cebula TA. Apoptosis induced by a cytopathic hepatitis A virus is dependent on caspase activation following ribosomal RNA degradation but occurs in the absence of 2'-5' oligoadenylate synthetase. Antiviral Res. 2004;63(3):153-66. DOI: 10.1016/j.antiviral.2004.02.004

30. Kocic G, Jevtovic T, Pavlovic D, Bjelakovic G, Kocic R. 290 A role of IFN- $\alpha$ in the double-stranded RNA degradation during Fas-ligand induced liver apoptosis and balance between proliferation and death. J Hepatol. 2006;44(06):S113-S. DOI: 10.1016/S01688278(06)80291-6

31. Koedoot E, Dévédec SL, Water BVD. Abstract 893: Systematic assessment of spliceosome components as drivers of breast cancer progression. Cancer Res. 2017;77(13 Supplement):893. DOI: 10.1158/15387445.AM2017-893

32. Quidville V, Alsafadi S, Goubar A, Commo F, Scott V, Pioche-Durieu C, et al. Targeting the deregulated spliceosome core machinery in cancer cells triggers mTOR blockade and autophagy. Cancer Res. 2013;73(7):224758. DOI: 10.1158/0008-5472.CAN-12-2501

33. Soto-Guzman A, Villegas-Comonfort S, Cortes-Reynosa P, Salazar EP. Role of arachidonic acid metabolism in Stat 5 activation induced by oleic acid in MDA-MB-231 breast cancer cells. Prostaglandins Leukotrienes \& Essential Fatty Acids. 2013;88(3):243-9. DOI: 10.1016/j. plefa.2012.12.003

34. Wang Z, Wang N, Han S, Wang D, Mo S, Yu L, et al. Dietary compound isoliquiritigenin inhibits breast cancer neoangiogenesis via VEGF/VEGFR-2 signaling pathway. Plos One. 2013;8(7):e68566. DOI: 10.1371/ journal.pone.0068566

35. Chun-Te C, Yi D, Hirohito Y, Jung-Mao H, Hsu-Ping $\mathrm{K}$, Hortobagyi GN, et al. Targeting the IKK $\beta / \mathrm{mTOR} /$ VEGF signaling pathway as a potential therapeutic strategy for obesity-related breast cancer. Mol Cancer Ther. 2012;11(10):2212-21. DOI: 10.1158/1535-7163. MCT-12-0180 